\title{
DREI AFRIKANISCHE STÄDTE: BAMAKO - KINSHASA - ZANZIBAR
}

\section{Maximilian Hendler}

Afrika, von dem hier nur der Teil südlich der Sahara betrachtet werden soll, ist ein polyzentrischer Kontinent. Ethnische Vielfalt und die Beeinflussung durch die ehemaligen Kolonialmächte ließen Schwerpunkte entstehen, die kaum mehr miteinander zu tun haben, als dass sie sich auf demselben Erdteil befinden - allen Aufrufen zur Einigkeit zum Trotz. Das gilt auf allen Gebieten, wirtschaftlich, gesellschaftlich und so auch auf dem Gebiet der populären Musik.

Gerade auf dem musikalischen Sektor werden Unterschiede feststellbar, die auf anderen Gebieten schwerer darzustellen sind. Während etwa in Accra, Johannesburg oder Nairobi eine stilistische Vielfalt herrscht, die nicht nur nie vereinigt wurde, sondern deren Vereinigung überhaupt nicht angestrebt wird, zeigen sich andere Zentren geschlossener und haben dementsprechend auch eine größere Wirkung auf ihre nähere und fernere Umgebung. Drei dieser Zentren sollen hier dargestellt werden: Bamako, die Hauptstadt der Republik Mali, Kinshasa, die Hauptstadt der Demokratischen Republik Kongo, und Zanzibar, die Hauptstadt der zur Republik Tanzania gehörigen Insel Unguja, die in Europa Zanzibar genannt wird.

Für jede dieser Städte steht eine CD, die in den Jahren 2004-2005 aufgenommen wurde, um die gegenwärtige Situation zu zeigen, die genauso wie in Europa oder den Vereinigten Staaten von elektrisch verstärkten bzw. elektronischen Instrumenten und den Raffinessen moderner Aufnahmetechnik geprägt ist. Umso deutlicher kommen die Unterschiede in der populären Musik der drei Hauptstädte zum Vorschein, die alles umfassen, was musikalische Relevanz haben kann: Themen, Instrumentierung, Einbettung in die musikalische Tradition usw. Dass jede CD charakteristische Tendenzen des jeweiligen Landes zeigt, ist dabei Voraussetzung. 


\section{Bamako: »Keme Burama« (Mandekalou 2004)}

Für diese CD stellte Ibrahima Sylla, der gewichtigste Produzent der Republik Mali, ein Ensemble zusammen, das als »All Star Band« der Mande-Musiker bezeichnet werden könnte, und nahm die Titel in der Nähe von Bamako selbst auf. Er vereinigte die besten Sänger und Musiker von Mali und Guinea, in denen sich das Erbe einer 700-jährigen Jeli-Tradition und das Image moderner Pop-Sänger mischt. »Jeli« oder »Jali« ist die Mande-Bezeichnung für eine spezifische Art von Sängern bzw. Sängerinnen, für die das deutsche Wort »Barde« am besten angebracht wäre. Im Gegensatz zu den europäischen Barden des Mittelalters ist diese epische Tradition im Sudan nie abgerissen. Das Ergebnis der Bemühungen Syllas ist die zeitgenössische Variante einer alten Überlieferung, die durch ihre fortgesetzte Modernisierung nie Gefahr lief, veraltet zu wirken. Vor allem die akustische Gitarre und die E-Gitarre haben sich einen festen Platz im Instrumentarium dieser Weltgegend erobert, ohne jedoch die traditionellen Musikinstrumente zu verdrängen.

Von den Interpreten dürfte der Sänger und Nkoni-Spieler Kasse Mady Diabaté in Europa durch die Veröffentlichungen Fode (1989) und Kela Tradition (1990) der bekannteste sein. Die Nkoni (andere Namen: Garaya, Hoddu, Khalam, Molo, Tidinit usw.) gehört zu einem heute nur mehr im Sudan gebrauchten Lautentyp, dessen Saiten nicht durch Pflockwirbel, sondern durch Spannbünde gehalten werden. Dazu ist ihr Hals nicht halbrund wie der aller anderen Lauten, sondern rund. Dieser Lautentyp lässt sich über Altägypten (Hickmannn 1961: 313) nach Altmesopotamien (Rashid 1984: 92) zurückverfolgen, wo er Ende des dritten Jahrtausends v. Chr. bei den Akkadern verwendet wurde. Gleichzeitig gehört die Laute vom Typ der Nkoni zu den Vorläuferinstrumenten des Banjos (Hendler 1991: 131-138). Durch elektrische Verstärkung kann sie sich bis heute neben den E-Gitarren halten. Es dürfte auf der Welt keine weitere populäre Musik geben, in der ein Instrument mit gut 4000jähriger Geschichte eine Rolle spielt.

Neben der Nkoni spielen auch die Kora oder Kalebassenharfe und das Xylophon im Ensemble tragende Stimmen. Die Kora wird von Mamadou Diabaté gespielt. Sie hat eine Kalebasse (ein vollreifer tropischer Kürbis) mit Felldecke als Resonator. Die 21 Saiten, die auf zwei Spielebenen verteilt sind, werden an einem verhältnismäßig dicken Stab angebracht, der durch zwei Löcher in die Kalebasse gesteckt ist. Die Verbindung der Saiten mit dem Resonanzkörper wird durch einen Kerbsteg hergestellt, der auch die Saiten in die richtige Spielposition bringt. Gespielt wird die Kora nur mit 
den Daumen der beiden Hände, während die anderen Finger die Kora an zwei Griffen halten, die links und rechts des Saiten tragenden Stabes befestigt sind. Das Xylophon (Mandingo: Balafon) hat 22 Stäbe und wird von Alkaly Camara gespielt, der es schon im Nationalballett »Djoliba«, das 1961 gegründet wurde, bediente. Nach der Überlieferung der Mande stammt die Urform des Balafons aus dem 13. Jahrhundert, wo sie der Jeli Faseke Kouyaté konstruierte.

Das Lied dreht sich um Almamy Samory Touré und seinen jüngeren Bruder Keme Burama (in anderen Quellen: Birama), die letzten Feldherrn der Mande, die im 19. Jahrhundert dem Vorrücken der Franzosen in den westsudanischen Gebieten einen ernsthaften Widerstand entgegensetzten. Eine andere Version desselben Liedes wurde in der Interpretation des MaliInstrumentalensemble (o. J.) veröffentlicht. Darin äußert sich ein weiterer Unterschied zum so genannten Westen. In der populären Musik Europas und Nordamerikas spielen heroische Themen keine Rolle. Anders verhält es sich in den ehemaligen Kolonialgebieten, wo der Kampf gegen die europäischen Eindringlinge erst mit der Befreiung vom kolonialen Joch ein Ende fand. Vor allem bei Ethnien mit intaktem Geschichtsbewusstsein, wie es die westsudanischen Völker durchweg sind, ist die Erinnerung an den Widerstand durchaus lebendig.

\section{Kinshasa: »Bombole Motema« (Mbilia Bel 2004)}

Die in Paris, aber ebenfalls von Syllart - der Plattenfirma von Ibrahima Sylla - aufgenommene CD, die Kinshasa gewidmet ist, bringt die neusten Einspielungen von Mbilia Bel, die im Begleittext als »Diva « bezeichnet wird. Ihr Alter ist nicht angegeben, doch dürfte sie um die 40 sein. Um ihre Art zu singen würdigen zu können, ist eine Bemerkung zur Struktur der BantuSprachen notwendig. Diese sind Tonsprachen, d.h. die Bedeutung eines Wortes hängt nicht nur von seinem Silbenverlauf $a b$, sondern auch davon, mit welchen Ton oder welcher Tonbewegung dieses Wort ausgesprochen wird. In der Sprachwissenschaft werden diese Töne als »phonologisch relevant « bezeichnet. Das bewirkt auch ohne musikalische Komponente eine verglichen etwa mit dem Deutschen - »melische« Kontur der gespochenen Sprache. Die verschiedenen Trommeln (»Sprechtrommeln«) und Holzidiophone, die in schwarzafrikanischen Ländern zur Wiedergabe der Sprache benützt werden, bedienen sich dieses Effekts. Sie geben die Sprache nicht im Sinn eines »Morsealphabetes « wieder, sondern sie ahmen die Töne und Tonverläufe der gesprochenen Sprache nach. 
Diese Sprachstruktur hat musikalische Konsequenzen. Träger der BantuSprachen können nicht beliebige musikalische Themen aus anderen Musikkulturen übernehmen, sondern zu jedem Text muss eine eigene Melodie geschrieben werden, die seinem Tonverlauf angemessen ist. Das ist der Grund, warum Musik dieses Erdteils eine nur ihm eigene Melodiekontur hat, es sei denn, dass englische, französische oder spanische Texte mit übernommen werden. Die Berichte, in denen frühe Missionare glaubten, auf die Melodien europäischer Kirchenlieder Bantu-Texte schreiben zu können und damit schallendes Gelächter ihrer Zöglinge ernteten, sind Legion. Am Anfang des Titels »Bombole motema« wechselt Mbilia Bel mehrmals zwischen gesungenem und gesprochenem Text. In diesen Partien ist die Struktur der Tonsprachen gut zu hören.

Im Übrigen pflegt Mbilia Bel einen außerordentlich melodiösen Gesangsstil, der in Zeiten, in denen der Rap in den westlichen Hitparaden einen hohen Stellenwert hat, geradezu befremdlich wirken muss. Sie hat dafür ein berühmtes Vorbild. In ihrem Gesang schwingt die Melodienseligkeit mit, die Lokanga La Ndju Pene Luambo Makiadi, kurz »Franco« genannt, mit dem 1956, also noch zur Zeit der belgischen Kolonialherrschaft, gegründeten Orchester O.K. Jazz in die populäre Musik der Demokratischen Republik Kongo (ehemals Zaire) einführte. Sänger und Orchester waren für drei Jahrzehnte Maßstab setzend in der Entwicklung der zeitgenössischen Musik. Darüber hinaus finden sich Elemente des von ihnen geprägten Stils in den meisten Nachbarländern, teilweise sogar darüber hinaus. Franco starb Ende der 1980er Jahre an AIDS, doch der von ihm geprägte Stil lebt weiter (CD-Veröffentlichungen seiner Musik befinden sich auf Div. 1993 und Franco et le T.P. O.K. Jazz 1990).

Welche Bedeutung das Wort »Jazz« für Franco und seine Musikerkollegen hatte, lässt sich heute nicht mehr ermitteln. In der Musik des Orchesters O.K. Jazz findet sich jedenfalls nichts, was in den 1950er Jahren und auch später unter diesem Begriff verstanden worden wäre. Soweit westlicher Einfluss vorhanden ist, kommt er primär aus Kuba. Immerhin war das Ensemble der wichtigste Träger der kongolesischen Rumba-Mode. Wieweit ältere Spuren aus Europa anzunehmen sind, wäre zu diskutieren, da diese Frage für große Teile des Kongo im Raum steht. Das Ensemblespiel von O.K. Jazz stützt sich stark auf autochthone Traditionen, die sich vor allem in Verzahnungseffekten äußern. Streckenweise macht es den Eindruck, als seien afrikanische Harfen- oder Lamellophontechniken auf Gitarren und Bass übertragen worden. Auch der Gesang Francos ist durch sein zwischen schmeichelnd und strahlend wechselndes Stimmtimbre und seine geschmeidige Phrasierungstechnik das Gegenteil dessen, was unter »Jazzgesang « im 
Sinn einer Billie Holiday oder Ella Fitzgerald verstanden wird. Jazz sollte in diesem Kontext wahrscheinlich signalisieren, dass sich die Musiker als »modern « verstanden, dass sie damit ihr Streben nach Anschluss an die »große Welt $«$ dokumentieren wollten.

\section{Zanzibar: Mohamed Ahmed - Mpenzi Wangu Hawezi« (Ikhwani Safaa Musical Club 2005)}

Bevor noch auf den Inhalt der CD eingegangen wird, die Zanzibar gewidmet ist, fällt ein formaler Unterschied gegenüber den anderen CDs auf, die sich mit afrikanischen Zentren beschäftigen. Während diese von einem Afrikaner initiiert sind, nahm für jene der Deutsche Werner Graebner die Titel auf. Das heißt nicht, dass die Musik dieser CD auf Zanzibar nicht geschätzt würde, doch die Strategien zur Plattenherstellung und Vermarktung sind den Bewohnern Zanzibars und im weiteren Sinn Tanzanias noch zu fern, als dass sie auf diesem Gebiet aktiv werden könnten. Hier erfüllt Werner Graebner eine Funktion, die der Musikethnologie herkömmlichen Sinnes gleichkommt.

Die CD enthält einen Querschnitt durch das heutige Schaffen des Ikhwani Safaa Musical Club, der seit 1905 in Zanzibar existiert und etliche bedeutende Musiker und Sänger der Insel hervorgebracht hat. Das Repertoire dieser Vereinigung umfasst heute noch einen Liedbestand aus einem Zeitraum von circa 70 Jahren, der von den Musikern und Sängern aktiv gepflegt wird. Damit ist ein weiterer Unterschied gegenüber den anderen CDs angesprochen. Nicht das »Neueste « wird angestrebt, sei es im Repertoire einer Sängerin oder in der Bildung eines Ensembles, sondern das Bewährte wird dargeboten, das zwar jederzeit um neue Kompositionen vermehrt werden kann, doch haben diese sich in den konventionellen Bahnen zu halten. Für Europäer wird dieser Effekt zwar durch die vordergründige Fremdheit der Aufnahmen unkenntlich gemacht, aber im Rahmen Afrikas ist dieser Unterschied signifikant.

Die Musik, die an den ostafrikanischen Küsten gepflegt wird, heißt »Taarab«. Es handelt sich um ein Wort aus dem Arabischen, dessen Grundbedeutung »Unterhaltung mit Musik« ist. Diese Bezeichnung wirft nicht nur ein Licht auf die Musik, sondern auch auf die Bevölkerung, die sie pflegt. Aus der andauernden Präsenz von arabischen und iranischen Händlern seit der Spätantike in den ostafrikanischen Küstengegenden, die oftmals einheimische Frauen nahmen, entstand eine orientalisch-afrikanische Mischbevölkerung mit einer bemerkenswerten Mischsprache und Mischkultur. Ihr Name 
leitet sich ab von arabisch »Sahel « = »Küste «, in der Mehrzahl »Sawahil « = »die Küsten«. Das Eigenschaftswort dazu lautet »swahili $«=» d e r$, die, das küstenländische«. Die Sprache dieser Swahilibevölkerung trägt den Namen »Kiswahili«. Ki- ist in den Bantu-Sprachen das Werkzeugpräfix. Wann sie sich formierte, ist nicht exakt feststellbar. Die ältesten Kiswahili-Texte stammen aus dem 13. Jahrhundert, doch zeigen sie bereits alle wesentlichen Merkmale der heute verwendeten Sprache. Die »Geburt « des Kiswahili ist also früher anzusetzen, wahrscheinlich parallel zum Prozess der Islamisierung.

In der grammatischen Struktur handelt es sich bei Kiswahili um eine Bantu-Sprache, die jedoch so stark mit arabischen und persischen Lehnworten überfremdet wurde, dass sie ein wesentliches Merkmal der Bantusprachen verlor - die Tonhöhen. Ihre musikhistorische Bedeutung liegt darin, dass ihre Sprecher frei sind, damit jede beliebige Melodie zu texten. Das ist ein wesentlicher Faktor, denn populäre Musik ist in Afrika wie im gesamten Tropengürtel in erster Linie Gesang. Ferner ist Kiswahili die bedeutendste überregionale Verkehrssprache in Ostafrika, die von Norden bis Süden verstanden wird und heute auch schon tief in das kontinentale Afrika hineingreift. Kiswahili-Texte finden somit einen Musikmarkt, dessen Größe von keiner anderen Sprache Ostafrikas erreicht wird. Das begünstigt rückwirkend wieder die Ausbreitung des Kiswahili, die auch in der Gegenwart weitergeht.

Die Entstehung des Taarab in Ostafrika hängt kausal mit dem KiswahiliIdiom zusammen. Es handelt sich um eine orientalische Musik mit KiswahiliTexten, die im Lauf der Zeit um indische und im späteren 20. Jahrhundert auch um lateinamerikanische Einflüsse bereichert wurde. Der indische Einfluss auf den Taarab geht weniger von der Musik der zahlreichen ostafrikanischen Inder aus, sondern von der Musik der indischen Filme, der Hindi Pictures (im heutigen Sprachgebrauch: Bollywood), die in Europa erst seit kurzem bekannt, im Orient und in Afrika jedoch allgegenwärtig sind. Westliche Moden im Taarab werden häufig über dieses Medium rezipiert und nicht direkt aus den Herkunftsländern, was sie für ein westliches Ohr kaum mehr erkennbar macht. Trotz aller Offenheit für modische Neuerungen ist der Taarab jedoch eine ernste Gattung. Das äußert sich unter anderem in der Existenz von Gruppen, die überhaupt keine »trivialen« Konzerte geben, sondern ausschließlich zu Hochzeiten, Beschneidungen und an den hohen islamischen Festtagen spielen.

Der Sänger von »Mpenzi Wangu Hawesi« (»Meine Geliebte ist krank«) ist Mohamed Ahmed, der 1940 geboren wurde. In den 1960ern war er einer der wichtigsten Sänger der Vereinigung, zog sich aber von der praktischen Musikausübung zurück, als er in den 1970ern ein Amt in der Inselverwaltung 
annahm. Er blieb jedoch einer der produktivsten Komponisten der Insel und singt seit seiner Pensionierung wieder. Die Melodie von »Mpenzi Wangu Hawesi « stammt aus den 1920ern und wurde mit verschiedenen Texten gesungen. Der Text der vorliegenden Aufnahme wurde um die Zeit von 1940 verfasst. Dieser Titel spiegelt das gesamte Repertoire des »lkhwani Safaa Musical Club« wieder. Dass die Vereinigung nicht nur Zanzibar betreut, sondern auch die Swahili im Ausland, führt dazu, dass ein Teil der Aufnahmen nicht auf der Insel, sondern in Dubai am Persischen Golf gemacht wurde. In den arabischen Ölförderländern sind viele Arbeitsmigranten aus den Swahililändern tätig.

Alle drei CDs geben Musik der Jahre 2004/2005 wieder, und die Aufnahmetechnik entspricht diesem Zeitraum. Andererseits pflegen sie eine Beziehung zur Tradition, die dem so genannten »Westen« völlig fremd ist. Am nächsten kommt der Kongo westlichen Gepflogenheiten. Das Faktum, dass ein Interpret einen Standard setzt, der von nachkommenden Generationen weitergeführt wird, findet sich sowohl in Europa als auch in den Vereinigten Staaten. Es handelt sich um eine ausgesprochen großstädtische Musik, die im Rahmen des Kongo und seines Umfeldes keinerlei ethnische Bindung mehr erkennen lässt, was in Afrika bis heute eher die Ausnahme als die Regel ist. Der Unterschied liegt darin, dass Franco einen den derzeitigen »westlichen « Moden entgegen gesetzten Standard setzte, der von Mbilia Bel weitergeführt wird. Die »repressive Toleranz « des Westens, die sich darin äußert, dass dem überwiegend jugendlichen Publikum in der populären Musik ein gerütteltes Maß an Protest in allen Formen zugestanden wird, muss sich in Afrika anderer Mittel bedienen, um Hörer zu finden.

Ein völlig anderes Bild bietet Zanzibar. Analoge Vereinigungen wie der »Ikhwani Safaa Musical Club« lassen sich in Europa zwar finden, doch spielen sie in der populären Musik heute keine Rolle. Ein wesentlicher Teil dieses Faktums liegt darin begründet, dass die Swahili-Populationen dem Islam angehören, der auf der arabischen Halbinsel, von wo sie ihn empfingen, ein ausgesprochen konservatives Profil hat. Neuerungen, die Europäer im Allgemeinen gar nicht bemerken, gibt es auch im Taarab, doch dürfen sie nicht so weit gehen, dass sie sich im musikalischen Gesamtverlauf störend bemerkbar machen.

In dieser Musik existieren Entwicklungstendenzen, wie sie in Europa bis in das 19. Jahrhundert hinein herrschten. Im Gegensatz zu Kinshasa handelt es sich um kleinstädtische Musik. Sie wird - wie auch ihr arabisches Vor bild - von Musikern getragen, die größtenteils einen anderen Brotberuf ausüben und die Arbeit im Taarab als Nebenbeschäftigung betreiben. Das 
Städtische liegt einerseits an der weit über ländliche Musikkapellen hinausgehenden Fülle der Instrumentengattungen und an dem Ausmaß an bewusster Theorie, mit welcher der Taarab gepflegt wird, andererseits an den im weitesten Sinn orientalischen Themen, welche die Kernsubstanz der Musik bilden.

Gänzlich ohne europäischen oder amerikanischen Vergleich sind die Verhältnisse bei den Mande im Westsudan. Im Europa gibt es keine Traditionen mehr, die sich auf das 13. Jahrhundert zurückführen lassen. Revivalismen aller Art kommen dafür nicht in Frage. Dabei beziehen sich die Modernisierungen keineswegs nur auf die Gitarre, die im 20. Jahrhundert in die Jeli-Musik hineingenommen wurde. Auch die Kora, die nach Meinung der Fachgelehrten ca. 300 Jahre alt ist, war zu ihrer Zeit eine Modernisierung. Zwar gehören die Mande ebenfalls dem Islam an, doch stehen in ihrer populären Musik nationalistische Tendenzen stärker im Vordergrund. Ihre Islamisierung erfolgte auch nicht von der arabischen Halbinsel aus, sondern von der spezifisch nordafrikanischen, arabisch-berberischen Mischbevölkerung, in der Separatismen aller Art knapp unter der Oberfläche liegen.

Auch hier handelt es sich insofern um städtische Musik, als ein derart hochkarätiges Ensemble heute nur in einem städtischen Zentrum zusammengestellt werden kann. Der ethnische Charakter des Unternehmens hebt es jedoch ab vom in Richtung Westen gehenden Internationalismus einer Mbilia Bel und dem orientalischen Internationalismus des »Ikhwani Safaa Musical Club«. Der Produzent Ibrahima Sylla wendet sich an die Volksgruppe der Mande, deren Traditionen ihm am Herzen liegen und denen er ein Denkmal setzten möchte. Die Zielgruppe dieser Emission sind nicht dörfliche oder städtische Gemeinwesen, sondern sie ist ethnisch bestimmt.

Mit dieser Auswahl wurden zwar wichtige Teile der afrikanischen populären Musik vorgestellt, die aber doch nur einen Ausschnitt des Musikschaffens im »schwarzen Kontinent « darstellen. Eine Fokussierung auf Dakar, Kampala und Luanda hätte andere Kombinationen musikalischer Elemente hervorgehoben, die genauso »afrikanisch « sind. Afrika ist - wie schon eingangs erwähnt - musikalisch ungemein vielgestaltig. Das »Afrikanische«, das gerade in der Musik vielfach gesucht wird, gibt es nicht. Daran ändert weder weißes Unverständnis etwas, für das die afrikanische Musik insgesamt entfernt und daher »gleich « klingt, noch schwarze Propaganda, die über alles hinweggeht, woran sich musikalische Unterschiede festmachen lassen. Afrika ist für den musikalisch Interessierten ein Dauerauftrag, der sich nie erschöpft und immer wieder Überraschungen zu bieten hat. 


\title{
Literatur
}

Hendler, Maximilian (1991). Banjo. Altweltliche Wurzeln eines neuweltlichen Musikinstruments (= Afro-Amerikanische Schriften 1). Göttingen: Edition Re.

Hickmann, Hans (1961). Ägypten (= Musikgeschichte in Bildern II: Musik des Altertums Lfg. 1). Leipzig: Deutscher Verlag für Musik.

Rashid, Subhi Anwar (1984). Mesopotamien (= Musikgeschichte in Bildern II: Musik des Altertums Lfg. 2). Leipzig: Deutscher Verlag für Musik.

\section{Diskographie}

Mali-Instrumentalensemble (o.J.). Erste Anthologie malinesischer Musik. Goldmedaille beim Panafrikanischen Kultur-Festival in Algier [Aufnahmen o.J.: B. Traore, A. Cormillot. Kommentar: M. M. Diabate]. Kassel u.a.: BärenreiterMusicaphon, BM 30SL2504.

Div. (1993). Roots of O.K. Jazz 1955-1956. Zaire Classics Vol. 3 [Zusammenstellung und Kommentar: Vincent Kenis]. Brüssel: Cram world, craw 7.

Franco et le T.P. O.K. Jazz (1990). Originalité. The Original 1956 Recordings of O.K. Jazz. London: RetroAfric, RETRO 2 CD.

Ikhwani Safaa Musical Club (2005). Zanzibara 1. 1905-2005. A Hundred Years of Taarab in Zanzibar [Aufnahmen 2004-2005: W. Graebner. Kommentar: R. Ramadhani]. Paris: Buda Records, 860118.

Kasse Mady (1989). Fode [Aufnahmen 1988: F. Perreard. Kommentar: H. Lee]. O.O.: Syllart/Mélodie, 38761-2.

Kasse Mady (1990). Kela Tradition [Aufnahmen o.J.: P. Braner]. New York: Stern's Africa , STCD 1034.

Mandekalou (2004). The Art and Soul of the Mande Griots [Kommentar: M. Hudson]. 0.0.: Syllart/Mélodie, 79663-2.

Mbilia Bel (2004). Belissimo [Aufnahmen o.J.: E. Colin.]. London: Stern's Africa, STCD 1098.

\begin{abstract}
This paper discusses the music of three African capitals, Bamako, Kinshasa and Zanzibar, by the example of three CDs published around 2004/2005. The pieces on the $C D$ representing the music of Bamako can only be understood on the background of the 700 years old tradition preserved by the Jelis, the bards of the Western Sudanic countries. The CD chosen as an example for the music of Kinshasa contains the newest creations by Mbilia Bel, who sings in the manner of Franco, the founder of the new music of Congo. The CD representing Zanzibar is dedicated to taarab, the music of the coasts of East Africa.
\end{abstract}

